\title{
Determination of Blood Lipid Concentrations among Type II Diabetic Patients in Aldwadmi Province, KSA
}

\section{Hanaa MAA*}

Sudan Academy of Sciences (SAS), Ministry of Higher Education and Research, College of Science, Shaqra University, KSA, Sudan

*Corresponding author: Hanaa MA Abdallah, Sudan Academy of Sciences (SAS), Ministry of Higher Education and Research, Khartoum, Sudan, College of Science, Shaqra University, KSA, Sudan, Tel: +966553149231; Email: hanaamahmoud94@gmail.com

\section{Research Article}

Volume 2 Issue 4

Received Date: July 25, 2018

Published Date: August 31, 2018

DOI: $10.23880 / \mathrm{mjccs}-16000172$

\section{Abstract}

Diabetes mellitus (DM) defined as a metabolic disorder of multiple etiology, characterized by chronic hyperglycemia with disturbances of carbohydrate, fat and protein metabolism resulting from defects in insulin secretion, insulin action, or both. WHO estimated that the number of people with DM worldwide in year 2000 was 177 million, this will increase to at least 336 million by the year 2030, this increase will be in the developing countries (approximately 75\% of all persons).

In this study we attempt to investigate the effect of DM on serum lipid profile among Saudis subjects with type 2 DM in Aldwadmi Province. Forty five Saudis diabetic patients were randomly selected from two hospitals (ALbejadia Hoapital, ALgimsh hospital) they were coming from different parts of Western of Alrhid. Their ages were between 18- 60 years, with a mean weight of $(70.20 \pm 11.203) \mathrm{Kg}$.

Thirty apparently healthy non diabetic people were chosen to serve as controls. Their ages were between (20-60) years and with a mean weight of (71.02 \pm 13.429$) \mathrm{Kg}$. All patients underwent a clinical and laboratory evaluation and answered standardized questionnaire. Glucose level, total cholesterol, TC, triglyceride, TG, low-density lipoprotein, LDL, and the high-density lipoprotein cholesterol, HDL were measured for all subjects.

The mean of fasting blood glucose (FBG) level of diabetic patients was significantly ( $p=0.001)$ higher than that of the non diabetic subjects and the same result was shown with random blood glucose (RBG). Significant difference was observed in lipid profile (TC ( $\mathrm{p}=0.03)$, TG $(\mathrm{p}=0.001)$ of patients compared to control subjects but the difference for HDL is not significant $(\mathrm{p}=0.74)$. The blood lipid concentrations and the blood glucose level are significantly higher among patients compared to the control subjects.

Keywords: Blood Lipid Concentrations; Diabetes Mellitus; Cardiovascular Disease; Lipid Metabolism; Chronic Hyperglycemia 


\section{Medical Journal of Clinical Trials \& Case Studies}

Abbreviations: DM: Diabetes Mellitus; CVD: Cardiovascular Disease; PA: Physical Activity; HDL: High Density Lipoprotein; LDL: Low Density Lipoprotein; TC: Total Cholesterol; TG: Triglyceride; HDL-C: High-Density Lipoprotein Cholesterol; SD: Standard Deviation; FBG: Fasting Blood Glucose; RBG: Random Blood Glucose; CETP: Cholesterol Ester Transport Protein.

\section{Introduction}

Diabetes mellitus (DM) is a disease mainly characterized by hyperglycemia, lipoprotein disorder and impairing of the oxidative metabolic pathways [1,2]. Diabetes, mostly the form of type 2 , has become both an economic and health problem globally [2]. The prevalence of diabetes among adult population worldwide is 285 million, and expected to increase to 439 million by 2030 [3]. Long term hyperglycemia is linked with the prolonged side effects of the disease which include serious destroy and the reduction of the efficiency of the cardiovascular system, eyes, kidney and nerves [2]. The main features of the disease are polyuria, polydipsia and weight loss even with the presence of the polyphagia, hyperglycemia, glycosuria, ketosis, acidosis and coma. Moreover, there are some symptoms usually connected with the disease, e.g. a false feeling of tiredness or sickness, irritation, more urination than usual, drinking more water and eating more food than normal meals, rising in the body temperature, headache, and itching and dryness of the skin [4]. Diabetes is characterized by chronic hyperglycemia and disturbances of carbohydrate, lipid and protein metabolism [5].

Diabetic patients may have some diseases related to the complication of the disease that include lipid metabolism disorder and cardiovascular disease (CVD) which may lead to serious morbidity and consequently to mortality [6]. These complications can be dramatically reduced by good diet regime and by adjusting the lipid concentrations in the blood circulation, medically or nutritionally, consequently these modifications may avoid the risk of (CDV) related diseases [7].

The triglceride-to-HDL cholesterol (TG/HDL) ratio has been investigated recently for various potential clinical uses in adult and paediatric populations, some studies showed positive associations with adverse cardiometabolic risk factor profiles, metabolic syndrome and prediction of incident diabetes or its complications $[8,9]$. High concentration of serumTG and low level of HDL cholesterol are the main features of the uncontrolled diabetes melitu [10,11]. This clearly shows the association of diabetes mellitus with the dyslipidemia which is usually appears parallelly with alteration in the blood glucose [12]. The acceleration of atherosis in diabetes mellitus may be due to high concentration of different types of lipids and extremely high synthesis of total cholesterol during hyperglycemia [13]. Lipid level, which is highly affected in diabetes mellitus, is one of the main causes in the occurrence of cardiovascular diseases [14-16]. Also, the alteration of the LDL oxidation is a crucial stage in the development of atherosclerosis [17].

\section{Diabetes mellitus in Saudi Arabia}

DM is spreading rapidly in Saudi Arabia because of the considerable change in the lifestyle of people in this country, because of the fast growth and development of the economy. High prevalence of the disease has been reported in the early studies conducted earlier in 1980s and more recent studies showed an even higher prevalence of DM in the Saudis $[18,19]$. The prevalence of type1 DM, type2 DM and impaired glucose tolerance in the total Saudi male population was $0.23 \%, 5.63 \%$ and $0.50 \%$, respectively, and in the total Saudi female population was $0.30 \%, 4.53 \%$ and $0.72 \%$ respectively. Differences were observed in the prevalence of diabetes mellitus and impaired glucose tolerance between the provinces. T2DM increased to $28.82 \%$ and $24.92 \%$ in males and females, respectively over the age of 60 years, while impaired glucose tolerance increased to $1.60 \%$ and $3.56 \%$.

High prevalence of diabetes mellitus worldwide is associated with lifestyle and aging population [20]. Many factors are etiology determined, namely, body composition, obesity habitual physical activity (PA) levels, diet and smoking, and all are studied for their effects on insulin resistance and T2DM. These etiological factors are considered to play a very clear role in the determination of type2 DM prevalence, especially during the last few decades [21]. More than 70\%of type2 diabetes cases appear to be associated with obesity and smoking [22]. Regular sport exercise and reduction of $5 \%$ of the body weight can decrease the rate of T2DM by $58 \%[23,24]$. Change in lifestyle and physical activities are the main factors in the prevention and control of the disease [25].

\section{Materials and Methods}

This study was conducted during March 2014 in Aldwadmi province, the largest city of the Alrig had it had an estimated total population of approximately million 


\section{Medical Journal of Clinical Trials \& Case Studies}

people, according to national population census in 2014, venous blood samples were obtained from people aged 18 years to 80 years. Blood samples were transferred to the central laboratory at Aljazeera private hospital for separation and to be analyzed by colorimetric method.

Forty five diabetic patients were randomly selected from two hospitals (ALbejadia Hospital, ALgimsh hospital) they were coming from different parts of Western villages of Alrhid. Their ages between 18- 60 years, a mean weight of $(70.20 \pm 11.203) \mathrm{Kg}$. Thirty apparently healthy, non diabetic people were chosen as controls. Their age between (20-60) years and mean of weight was (71.02 \pm 13.429$) \mathrm{Kg}$.

All patients underwent a clinical and laboratory evaluation and answered a standardized questionnaire. Blood samples for glucose level, total cholesterol, triglyceride, low-density, and lipoprotein high-density cholesterol were measured for each subject (diabetic and non-diabetic).

Information's were taken from each subject about, treatment, family history of diabetes and other diseases and complication. Both Patients and control subjects were interviewed at the hospital.

The following list of equipment and consumables were used to perform different experiments and measurements; COBAS INTEGRA $\mathbf{4 0 0}$ plus which is a fully automated, random access, sample discrete, multianalyte analyzer, centrifuge EBA20 from Germany, Plain Blood containers, Pipettes $(10 \mu \mathrm{L}-1000 \mu \mathrm{L})$ and disposable tips, fluoride blood containers, and storage tubes.

Ready to use reagents in a unique reagent carrier provided by Roche to fit COBAS INTEGRA 400 plus were used for the measurement of glucose concentration and lipid profile (TG, TC, HDL and consequently the values of LDL were calculated)

After approximately 12 hours fasting period $5 \mathrm{ml}$ of venous blood in the dry sterile syringe were collected from each subject (patients and controls). The blood was divided into 2 tubes. $2 \mathrm{ml}$ was mixed with floride oxalate in a fluoride blood container for blood glucose measurement and $3 \mathrm{ml}$ were allowed to clot and immediately centrifuged at 2000 r.p.m for 10 minutes and sera was used for the lipid profile test.

The method used as described earlier with Sharma [26].

This method was used (COBAS INTEGRA 400 plus) to determine the concentrations of glucose, triglycerides, cholesterol, high density lipoprotein (HDL), low density lipoprotein (LDL)

\section{Statistical Analysis}

All data were processed using the Statistical Package for the Social Sciences (SPSS version16, Chicago, USA software).The differences between the groups were tested for significance by student's t-test. Data were expressed as the mean \pm SD. Differences considered statistically significant if $\mathrm{p}$-values $<0.05$.

\section{Results}

This study was a hospital based, cross sectional study to compare the blood glucose level and lipid profile concentrations in diabetic and non-diabetic subjects. To perform this, a comparison analysis was done to see if there is any difference between controls and patients. The results of this study covered forty five known diabetic patients and thirty healthy (non-diabetic) subjects (controls). Data collected from both diabetic patients and control subjects from Albjadia hospital and Aljimsh clinic Table 1. Represents the mean age and weight for both patients and control subjects. Plasma glucose level and serum lipid profile (total cholesterol (TC), triglyceride (TG), High-density lipoprotein cholesterol (HDL-C), were measured. The mean and standard deviation (SD) of all parameters were calculated.

\begin{tabular}{|c|c|c|c|c|}
\hline Characteristic & $\begin{array}{c}\text { Mini } \\
\text { mum }\end{array}$ & $\begin{array}{c}\text { Maxi } \\
\text { mum }\end{array}$ & Mean & $\begin{array}{c}\text { Std. } \\
\text { Deviation }\end{array}$ \\
\hline Patients' age (YR) & 18 & 70 & 44.47 & 15.811 \\
\hline $\begin{array}{c}\text { Patients' weight } \\
\text { (Kg) }\end{array}$ & 39 & 88 & 67.41 & 14.009 \\
\hline $\begin{array}{c}\text { Control subjects } \\
\text { age (YR) }\end{array}$ & 18 & 58 & 43.13 & 10.868 \\
\hline $\begin{array}{c}\text { Control subjects } \\
\text { weight (Kg) }\end{array}$ & 50 & 82 & 66.27 & 10.140 \\
\hline
\end{tabular}

Table 1: Age and Weight of Diabetic Patients and Control Subjects (Mean $\pm \mathrm{SD}$ ).

\section{Blood Glucose Level}

The mean of fasting blood glucose (FBG) level of diabetic patients was significantly $(\mathrm{p}=0.001)$ higher than that of the non diabetic subjects as shown in table 2 . The mean of random blood glucose (RBG) level of diabetic patients was significantly $(\mathrm{p}=0.001)$ higher than that of the non diabetic subjects as shown in table 2 , and figure 2 . 


\section{Medical Journal of Clinical Trials \& Case Studies}

\begin{tabular}{|c|c|c|c|}
\hline $\begin{array}{c}\text { Measurement } \\
(\mathrm{mg} / \mathrm{dL})\end{array}$ & $\begin{array}{c}\text { Diabetic } \\
\text { patients } \\
(\text { Mean } \pm \text { SD) }\end{array}$ & $\begin{array}{c}\text { Non-Diabetic } \\
\text { subjects } \\
\text { (Mean } \pm \text { SD) }\end{array}$ & p- value \\
\hline FBG & $171.00 \pm 64.42$ & $96.33 \pm 14.34$ & 0.01 \\
\hline RBG & $254.83 \pm 83.90$ & $143.73 \pm 63.73$ & 0.01 \\
\hline TC & $27.91 \pm 65.60$ & $4.57 \pm 01.22$ & 0.03 \\
\hline TG & $67.18 \pm 10.38$ & $45.87 \pm 10.21$ & 0.001 \\
\hline HDL & $92.00 \pm 57.75$ & $82.37 \pm 66.57$ & 0.74 \\
\hline
\end{tabular}

\section{Lipid Profile: TC, TG, and HDL Concentrations}

Total Cholesterol mean level value in diabetic patients was significantly higher than the mean serum of non diabetic subjects $(\mathrm{p}=0.03)$.

The mean value of triglycerides in diabetic patients was significantly higher than the mean value of non diabetic subjects $(\mathrm{p}=0.001)$.

The mean value of serum HDL-Cholesterol in diabetic

Table 2: Levels of Glucose and Serum Lipid Profile in Diabetic Patients and Control Subjects. patients was higher than the mean value of non diabetic subjects, but, this difference was not significant $(p=0.74)$. As shown in table 2 and figure 1.
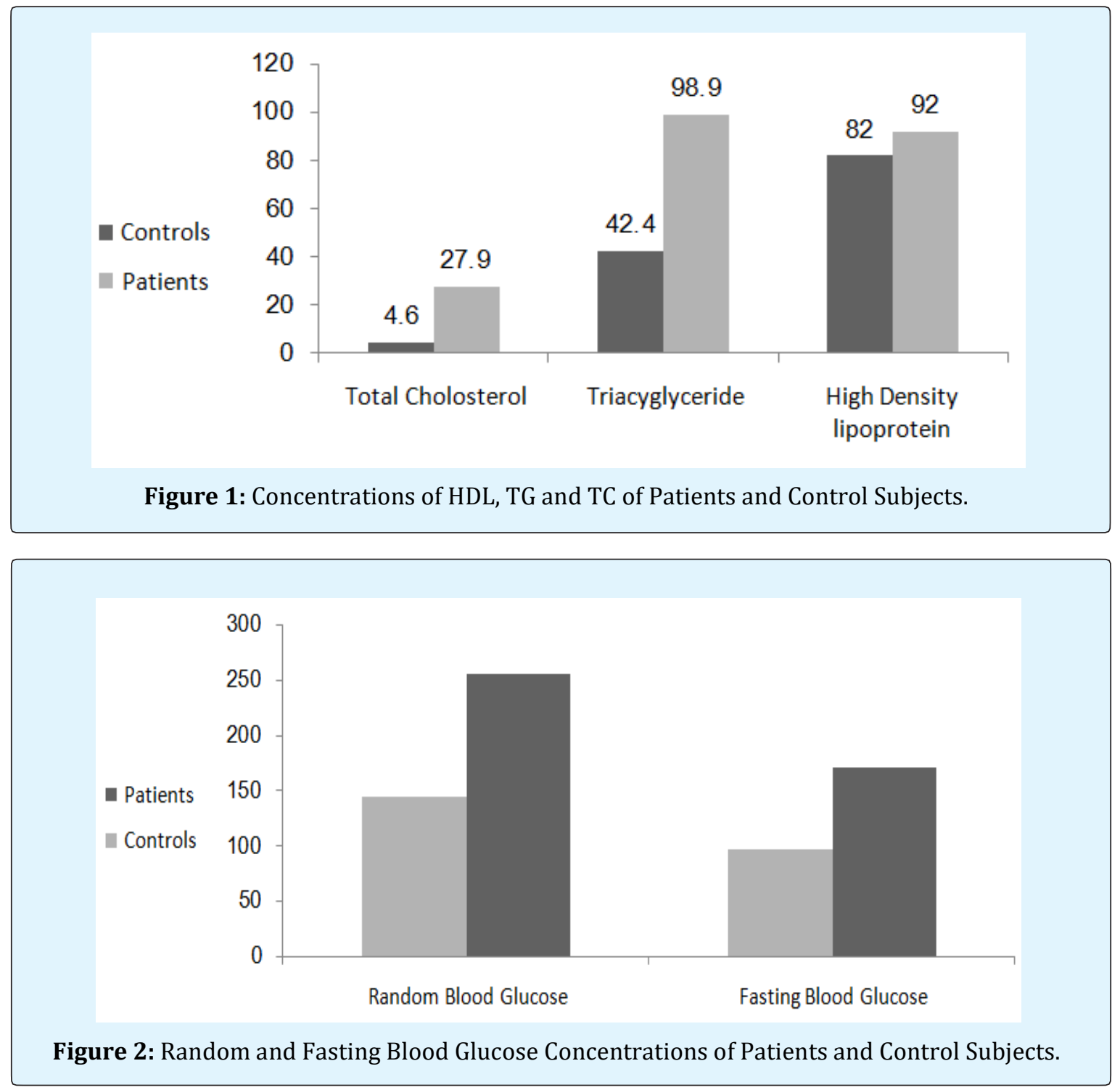


\section{Medical Journal of Clinical Trials \& Case Studies}

\section{Discussion}

The prevalence of T2DM and its complications is dramatically elevated in the Kingdom of Saudi Arabia (KSA) paralarly with the increase in the disease rate in different parts of the world as reported by the WHO [27].

The main features related with the disease in KSA are the obesity, very little physical activity (PA) and markedly change in people behavior and lifestyle. These factors contribute negatively to the already fragile situation and lead to serious complications. One of the most important causes of the complications is the abnormalities in lipids profile in the present study; we determined the prevalence of lipid profile. Furthermore, healthcare standards and patients' knowledge among Saudi diabetic patients are also studied.

In this study diabetic patients have abnormal level in the mean of fasting blood glucose compared with non diabetic subjects. A significant difference was observed $(p=0.001)$, the same result $(p=0.0 .001)$ was found between the mean of random blood glucose level (RBG) in patients compared to control subjects. High levels of blood glucose of diabetic patients due to lack of or resistance to insulin, the same results were previously reported [28-30]. These studies concluded that the fasting blood glucose level is also elevated and this indicates poor control of DM. As it is well known the main feature of DM is a persistant elevation of glucose in the blood (hyperglycemia) and this abnormality is associated with many metabolic abnormalities [31].

Impairing of lipid and lipoprotein metabolism are very common in the diabetic patients because of the effects of insulin lack and insulin resistance on the main enzymes act on the lipid metabolism [32]. Elevation of glucose level and the abnormality of insulin level and insulin resistance may lead to abnormalities in lipoprotein levels in the plasma and hyperinsulinaemia and consequently with the development of atherosclerotic vascular complications in diabetic population $[33,34]$.

Recent study provides evidence to suggest that lipid variables are associated with each other in T2DM patients among the population of Kishanganj, India [35]. The result of this study showed significant increased levels of total cholesterol $(p=0.03)$ in diabetic patients compared to non-diabetic subjects, this increase it may be due to an increase in the plasma concentration of VLDL and LDL, which may be due to increased hepatic production of
VLDL or decreased removal of VLDL and LDL from the circulation [36]. Significantly higher levels of triglycerides $(p=0.001)$ in Saudi diabetic patients may due to overproduction of VLDL lead to increased plasma levels of triglyceride which, via an exchange process mediated by cholesterol ester transfer protein (CETP), result in lower levels of high density lipoprotein HDL-cholesterol, also may be due to insulin deficiency which results faulty glucose utilization causes hyperglycemia and mobilization of fatty acids from adipose tissue.

In diabetes blood glucose is not utilized by tissue resulting in hyperglycemia which, consequently may lead to mobilization of fatty acids from adipose tissue for energy purpose and excess fatty acid is accumulated in the liver, unused fatty acids are usually converted to triacylglecerides (TGs) [37]. The most common abnormalities of lipid profile were a multi factorial complication of increased level of TGs (VLDL-TG), low clearance of TG-rich lipoproteins and extremely low highdensity lipoproteins HDL-C concentration [38]. Nonsignificant higher concentration of HDL $(p=0.74)$ in diabetic patients compared to non-diabetic subjects and decreased HDL cholesterol concentration would be due to TG combination with cholesterol ester transfer protein and high level of the TG lipase activity [39]. Inspite of the production of HDL particles in the hepatic tissues, a very big part of them are produced from the resident particles of TG-rich lipoproteins as they are breaking down.

This metabolism is usually impaired in DM, decreasing the formation of HDL-C from this source, a protein called cholesterol ester transport protein (CETP) transports cholesterol ester away from HDL particles in exchange for TG from VLDL particles. This transport decreases plasma HDL-C [40]. Lipid levels affected with glucose levels because carbohydrates and lipid metabolism are interrelated to each other if there is any disorder in carbohydrate metabolism will lead to disorder in lipid metabolism so there is a high concentration of cholesterol and triglycerides and due to this there is a reduction in HDL cholesterol levels because insulin resistance with or without hyperglycemia is associated with qualitative changes in the lipid profile [41]. These findings agreed with many researchers [42-44]. The prevalence and severity of dyslipidemia was associated with T2DM control, an early identification of dyslipidemia as a wellknown amendable risk factor of CVD is important for the implementation of therapy for effective prevention and management [45]. 


\section{Medical Journal of Clinical Trials \& Case Studies}

\section{Conclusions}

Highly significant differences in mean concentrations of TG and TC between patients and controls but the difference was not significant for HDL. Fasting blood glucose concentration among patients was significantly higher than random blood glucose of the control subjects. Measurement of serum lipid profile would be introduced to the management plan of diabetes mellitus.

\section{Acknowledgement}

My gratitude is to my colleages at the Department of Biology, College of Science, University of Shaqra, KSA, for their valuable assistance to perform this research project for the students. We are greatly indebted to staff of the two hospitals (ALbejadia Hoapital, ALgimsh hospital) for their valuable assistance in collection of blood samples. The financial support provided by Shaqra University, the key of success of this study, is highly appreciated.

\section{References}

1. Scoppola A, Montecchi FR, Meziinger G, Lala A (2001) Urinary mevaloate excretion rate type 2 diabetes: role of metabolic control. Atherosclerosis 156(2): 357361.

2. Wild S, Roglic G, Green A, Sicree R, King H (2004) Global Prevalence of Diabetes: Estimates for the year 2000 and projections for 2030. Diabetes Care 27(5): 1047-1053.

3. Shaw JE, Sicree RA, Zimmer PZ (2010) Global estimates of the prevalence of diabetes for 2010 and 2030. Diabetes Res Clin Pract 87(1): 4-14.

4. Young M, Richard M, Jeff M (2000) Managing type 2 diabetes. The journal of the American Medical Association. 288 JAMA 283.

5. Ozder A (2014) Lipid profile abnormalities seen in T2DM patients in primary healthcare in Turkey: a cross-sectional study. Lipids Health Dis 13: 183.

6. Harris M I (1998) Diabetes in America: epidemiology and scope of problem. Diabetes care (Suppl 3): C11C14.

7. Jonsson B, Cook J R, Pedersen T R (1999) The costeffectiveness of lipid lowering in patient with diabetes result from the $4 \mathrm{~s}$ trial. Diabetolgia 42(11): 1293-1301.

8. He S, Wang S, Chen X, Jiang L, Li L, et al. (2012) Higher ratio of triglyceride to high high-density lipoprotein cholesterol may predispose to diabetes mellitus: 15year prospective study in a general population. Metabolism 61(1): 30-36.

9. Zoppini G, Negri C, Stoico V, Casati S, Pichini I, et al. (2012) Triglyceride-high density lipoprotein cholesterol is associated with microvascular complications in type 2 diabetes mellitus. Metabolism 61(1): 22-29.

10. De Man, FH Caberzas MC, Van Barling HH, Erkelens DW, Debruin TW (1996) Triglyceride -rich lipoprotein in non -insulin dependent diabetes mellitus: post -prandial metabolism and relation to premature atherosclerosis. Eur J Clin invest 26(2): 89-108.

11. Barrett-connor E, Wingard DL (1983) Sex differential in ischemic heart disease mortality in diabetic a prospective population-based study. Am J Epidemiol 118(4): 489-496.

12. Kreisberg RA (1998) Diabetic dyslipidimia. Am J Cardiol 82(12A): 67U-73U; 85U-86U.

13. Bennion LJ, Grundy SM (1977) Effect of Diabetes mellitus on cholesterol metabolism in man. New Engl J Med 296(24): 1365-1371.

14. Betteridge DJ (1994) Diabetic dyslipidemia. Am J Med 96(6A): 25S-31S.

15. Kamata K, Yamashita K (1999) Insulin resistance and impaired endothelium dependent renal vasodilatation in fructose-fed hypertensive rats. Res Commun Mol Pathol Pharmacol 103(2): 195-200.

16. Kamata K, Kanie N, Inose A (2001) Mechanisms underlying attenuated contractile response of aortic rings to noradrenaline in fructose-fed mice. Eur J Pharmacol 428(2): 241-249.

17. Felmeden DC, Spencer CG, Blann AD, Beevers DG, Lip GY (2003) Low-density lipoprotein subfraction and cardiovascular risk in hypertension: Relationship to endothelial dysfunction and effects of treatment. Hypertension 41(3): 528-533. 


\section{Medical Journal of Clinical Trials \& Case Studies}

18. Bacchus R, JL Bell, M Madkour, B Kilshaw (1982) The prevalence of diabetes mellitus in male Saudi Arabs. Diabetologia 23(4): 330-332.

19. Abu-Zeid HAH, Al-Kassab ASK (1992) Prevalence and health-care features of hyperglycaemia in semiurbanrural communities in southern Saudi Arabia. Diabetes care 15(4): 484-489.

20. Hux J, Tang M (2003) Patterns of prevalence and incidence of diabetes. In: Hux J, Booth G, Slaughter P, Laupacis A, eds. Diabetes in Ontario: An ICES Practice Atlas. Toronto, ON: Institute for Clinical Evaluative Sciences 1.1-1.18.

21. Winfred $M$, Markus N, Hoffmann MM, Dietmar $N$, Boehm OB, et al. (2004) G(-30) A polymorphism in the pancreatic promoter of the Glucokinase Gene Associated with Angiographic coronary Artery Disease and type 2 Diabetes Mellitus. Circulation 109(23): 2844-2849.

22. Anderson JW, Kendall CW, Jenkins D J (2003) Importance of weight management in type 2 diabetes: review with meta-analysis of clinical studies. J Am Coll Nutr 22(5): 331-339.

23. Wing RR, Koeske R, Epstein LH, Nowalk MP, Gooding W, et al. (1987) Long-term effects of modest weight loss in type II diabetic patients. Arch Int Med 147(10): 1749-1753.

24. WHO. World Health Organization Prevention of diabetes mellitus. Geneva (1994) (WHO Technical Report Series, No. 844).

25. Sharma BK (2001) Instrumental Method of chemical Analysis. 20th (Edn.), Book Code No: CH3-20/ INDIA. Prakashan Media Ltd, pp: 45-44.

26. WHO, World Health Organization Consultation. Definition, diagnosis and classification of diabetes mellitus and its complications, part 1: World Health Organization. Diagnosis and classification of diabetes mellitus, Geneva (1999).

27. Richard M Bergenstal, Mary Johnson RN, Margaret A Powers, Alan Wynne, Aleksandra Vlajnic, et al. (2008) Adjust to Target in Type2 Diabetes, Comparison of a simple algorithm with carbohydrate counting for Adjustment of mealtime insulin glulisine. Diabetes Care 31(7): 1305-1310.
28. Abdelgadir M (2006) Clinical and Biochemical Features of adult Diabetes Mellitus in Sudan. Uppsala Dissertation from Faculty of Medicine.

29. Elbagier MN (1995) Prevalence of Diabetes Mellitus in Sudan. Uppsala Sweden Uppsala University.

30. Pari L, Latha M (2002) Effect of Cassia auriculata flowers on blood sugar levels, serum and tissue lipids in streptozotocin diabetic rats. Singapore Med J 43(12): 617-621.

31. (1999) Glucose tolerance and mortality. Comparism of WHO and American Diabetes Association diagnostic criteria. The DECODE study group. European Diabetes Epidemiology Group. Diabetes Epidemiology: Collaborative analysis of Diagnostic criteria in Europe. Lancet 354(9174): 617-621.

32. Simons LA, Simons J, Friedlander T, McCallum, J (2001) Cholesterol and other lipids predict coronary heart disease and ischemic stroke in the elderly, but only in those below 70 years. Atherosclerosis 159(1): 201-208.

33. United Kingdom Prospective Diabetes Study Group (2000) Association of glycemia with macro and microvascular complication of type2 diabetes (UK PDS 35). Brit Med J 321: 405-412.

34. Manisha M, Rina K, Arati M (2015) Prevalence of dyslipidemia in patients with type 2 diabetes mellitus: a hospital based study in Kishangani, India. Int J Res Med Sci 3(12): 3691-3697.

35. Ganong WF (2003) Review of Medical Physiology. $21^{\text {st }}$ (Edn.), Endocrine Function of pancreas \& Regulation of Carbohydrate Metabolism.

36. Shih KC, Kwak CF, Hwa CM, Hsiao LC, Li SH, et al. (1997) Acipimox attenuates hypertriglyceridemia in dyslipidemic noninsulin dependent diabetes mellitus patients without perturbation of insulin sensitivity and glycemic control. Diabetic Res clin Pract 36(2): 113-119.

37. Gowri MS, Westhuyzem D, Bridges SR, Anderson J W (1999) Deceased protection by HDL from poorly controlled type2 diabetic subjects against LDL oxidation may be due to the abnormal composition of HDL. Arteriosclerosis, Thrombosis, and Vascular Biology 19: 2226-2233. 


\section{Medical Journal of Clinical Trials \& Case Studies}

38. BrunzellJ D, Hokanson JE (1999) Dyslipidemia of central obesity and insulin resistant. Diabetes Care 22(3): c10-c13.

39. Hu FB, Willett WC (2002) Optimal diets for prevention of coronary heart disease. JAMA 288(20): 2569-2578.

40. Lamarche B, Tchernof A, Mauriege P, Cantin B, Dagenais GR, et al. (1998) Fasting insulin and apolipoprotein B levels and low-density lipoprotein particle size as risk factors for ischemic heart disease. JAMA 279(24): 1955-1961.

41. Carr MC, Kim KH, Zambon A, Mitchel ES, Woods NF, et al. (2000) Changes in LDL density across the menopausal transition. J Investigational Med 48(4): 245-258.
42. Mathews KA, Wing RR, Kuller LH, Meilahn EN, Plantinga P (1994) Influence of the perimenopause on cardiovascular risk factors and symptoms of middle-aged healthy women. Arch Int Med 154(20): 2349-2355.

43. Berestein FG, Bengtson C, Herbert PN (1993) Differences in LDL Subfractions and apolipoproteins in Premenopausal and Postmenopausal Women. J Clin Endocrinol Metab 40: 17-30.

44. Pandya H, Lakhani JD, Dadhania J, Trivedi A (2012) The prevalence and pattern of dyslipidemia among type 2 diabetic patients at rural based hospital anGuarat, India. J Clin Prac 22(12): 36-44.

45. Tagoe DNA, Amo Kodieh P (2013) Type 2 diabetes mellitus influence lipid profile of diabetic patients. Ann Biol Res 4(6): 88-92. 